\title{
BMJ Open Ethnographic investigation of patient- provider communication among African American men newly diagnosed with prostate cancer: a study protocol
}

\author{
Nynikka R Palmer (D) , 1,2,3 Janet K Shim, ${ }^{4}$ Celia P Kaplan, ${ }^{3,5}$ Dean Schillinger, ${ }^{1,2}$ \\ Sarah D Blaschko, ${ }^{6}$ Benjamin N Breyer, ${ }^{7,8}$ Rena J Pasick ${ }^{3,5}$
}

To cite: Palmer NR, Shim JK, Kaplan CP, et al. Ethnographic investigation of patient-provider communication among African American men newly diagnosed with prostate cancer: a study protocol. BMJ Open 2020;10:e035032. doi:10.1136/ bmjopen-2019-035032

- Prepublication history for this paper is available online. To view these files, please visit the journal online (http://dx.doi org/10.1136/bmjopen-2019035032).

Received 16 0ctober 2019 Revised 02 June 2020 Accepted 25 June 2020
Check for updates

(C) Author(s) (or their employer(s)) 2020. Re-use permitted under CC BY-NC. No commercial re-use. See rights and permissions. Published by BMJ.

For numbered affiliations see end of article.

Correspondence to Dr Nynikka R Palmer; nynikka.palmer@ucsf.edu

\section{ABSTRACT}

Introduction In the USA, African American men bear a disproportionate burden of prostate cancer (PCa) compared with all other groups, having a higher incidence and mortality, poorer quality of life and higher dissatisfaction with care. They are also less likely to receive guideline-concordant treatment (eg, undertreatment of aggressive disease). Inadequate patient-provider communication contributes to suboptimal care, which can be exacerbated by patients' limited health literacy, providers' lack of communication skills and time constraints in low-resource, safety net settings. This study is designed to examine the communication experiences of African American patients with PCa as they undertake treatment decision-making.

Methods and analysis Using an ethnographic approach, we will follow 25 African American men newly diagnosed with PCa at two public hospitals, from diagnosis through treatment decision. Data sources include: (1) audiorecorded clinic observations during urology, radiation oncology, medical oncology and primary care visits, (2) field notes from clinic observations, (3) patient surveys after clinic visits, (4) two in-depth patient interviews, (5) a provider survey, and (6) in-depth interviews with providers. We will explore patients' understanding of their diagnoses and treatment options, sources of support in decisionmaking, patient-provider communication and treatment decision-making processes. Audio-recorded observations and interviews will be transcribed verbatim. An iterative process of coding and team discussions will be used to thematically analyse patients' experiences and providers' perspectives, and to refine codes and identify key themes. Descriptive statistics will summarise survey data.

Ethics and dissemination To our knowledge, this is the first study to examine in-depth patient-provider communication among African American patients with $\mathrm{PCa}$. For a population as marginalised as African American men, an ethnographic approach allows for explication of complex sociocultural and contextual influences on healthcare processes and outcomes. Study findings will inform the development of interventions and initiatives that promote patient-centred communication, shared decisionmaking and guideline-concordant care. This study was approved by the University of California San Francisco and the Alameda Health System Institutional Review Boards.
Strengths and limitations of this study

- Potentially the first study to examine in-depth patient-provider communication among low-income African American patients with prostate cancer.

- Longitudinal, multimodal qualitative approaches and informants, and quantitative data will facilitate a more holistic perspective and yield a rich understanding of the experiences of African American patients with prostate cancer.

- Providers may demonstrate an increased awareness in communicating with patients as a result of being observed and interviewed, as well as patients interacting with providers.

- Recruitment from two safety net settings and qualitative study design may limit generalisability of findings.

- This comprehensive view will inform intervention development, in which community stakeholders will be engaged as cocreators (eg, collaborators) for maximum cultural fit and impact.

\section{INTRODUCTION}

High-quality cancer care occurs in a responsive healthcare system, where providers practise patient-centred communication and patients are informed and engaged. ${ }^{1}$ In such a context, each patient can expect to receive the most appropriate treatment for his condition and personal circumstances. This ideal, however, is often out of reach for one of the most marginalised groups in US societyAfrican American men, who also experience a disproportionate burden of prostate cancer (PCa).

African American men have an approximately $60 \%$ higher incidence, are more likely to be diagnosed with aggressive disease and have more than twice the mortality due to PCa compared with White men in the USA. ${ }^{2-4}$ Among the causes of excess mortality is failure to receive guideline-concordant treatment, in which men do not receive definitive treatment 
(eg, surgery and radiation) when it would be most beneficial (undertreatment). ${ }^{5-7}$ In addition, overtreatment of low-risk disease (therapies that may not be beneficial yet have serious side effects) contributes to poorer quality of life. These practices are at odds with at least three of the US National Academy of Medicine's (formerly the Institute of Medicine) six indicators of quality of care: (1) patient-centred communication and shared decisionmaking; (2) healthcare aligned with patients' needs, values and preferences; and (3) evidence-based medical decisions. $^{8}$

The nature and course of PCa and the array of treatment options present daunting communication challenges for patients and providers. First, neither surgery nor radiation has been found to be superior for survival. ${ }^{9}$ Second, national PCa treatment guidelines (eg, the National Comprehensive Cancer Network) call for risk stratification of disease aggressiveness as low, intermediate and high, ${ }^{10}$ and clinicians are advised to consider the combination of life expectancy and risk in differentiating 'appropriate' from 'inappropriate' treatment. ${ }^{1011}$ This information is then interpreted by patients and their families based on their own perspectives and priorities, as well as their histories of illness and healthcare. All of this can be extraordinarily difficult for patients to grasp and navigate, particularly in the face of multiple and often mutually exclusive treatment options. ${ }^{12}$

Furthermore, while patient-centred risk stratification aims to tailor treatment options to patient characteristics, aspects of patients' lives well beyond observable 'risk factors' can be critically relevant. Importantly, men with low-risk PCa (which may never be life-threatening) can be told that they have cancer which does not require immediate treatment. This means that the tests show their cancer is currently not life-threatening (unlikely to spread) such that they can avoid invasive treatment if offered 'active surveillance' (regular monitoring with periodic biopsies, prostate-specific antigen (PSA) blood tests and other testing). Patients considering this option must fully grasp the fact that they have a cancer that does not require immediate treatment, but since the cancer can change a protocol of periodic, uncomfortable procedures (eg, biopsies) must be followed. For some African American men, this taps into historical mistrust of the healthcare system and fear that a disease will be intentionally untreated, as in the well-known example of the Tuskegee study of untreated syphilis. ${ }^{13}{ }^{14}$ Alternatively, opting for active treatment (eg, surgery or radiation) can result in impotence or incontinence, a prospect which evokes considerable distress. ${ }^{15}$ Various studies have noted that a PCa diagnosis and its treatment can be perceived as a threat to African American men's sense of masculinity, impacting communication and well-being. ${ }^{16-19}$ Patient comprehension and integration of these complex issues are essential as only he can know which configuration best fits his individual needs and preferences.

Unfortunately, disparities in healthcare in general are most pronounced among patients with low health literacy, partially explaining racial disparities in some health outcomes. ${ }^{20}$ Specifically in PCa, African American men with low health literacy have worse outcomes, including greater distress ${ }^{21}$ and higher PSA level at diagnosis (indicative of more advanced disease),${ }^{22}$ which may reflect larger socioeconomic (eg, literacy, income, education) differences. Previous studies have noted significant disparities in PCa care among lower income and uninsured African American men, ${ }^{23}{ }^{24}$ and in low-resource settings (eg, public hospitals and other safety net systems), ${ }^{25-27}$ where time constraints exacerbate communication barriers and degrade quality of care. Health literacy of patients with PCa impacts their ability to engage in shared decisionmaking. ${ }^{28}$ Low health literacy level can limit patient understanding of complex treatment information and potential side effects, and can hinder patient participation in the decision-making process. ${ }^{29}$ Importantly, patients' individual preferences and priorities must be communicated, enacted and facilitated in the decision-making process, as one size does not fit all in shared decision-making. ${ }^{30-34}$ These challenges are further complicated by evidence that patients with PCa tend to rely heavily on providers' recommendations, ${ }^{3536}$ and PCa specialists tend to favour the treatment they deliver. ${ }^{37}$ Moreover, an African American man seeking cancer treatment might wonder which poses a greater threat to him-medical care, or the disease itself. In this context and with limited resources, the path to effective communication between safety net providers and African American patients with PCa is fraught.

We seek to decode the constellation of societal, institutional and individual forces through an in-depth exploration of African American men's communication experiences in PCa care, as he moves from prostate biopsy to cancer diagnosis and treatment decision-making. Our research is broadly informed by the theory of cultural health capital (CHC) which elucidates the influence of fundamental social inequalities on clinical interactions, complicating patient-centred care and intensifying inequality. ${ }^{38}$ Recognising the power of culture and social context that people implicitly draw on to make sense of their world, ${ }^{39}$ including contending with illness and healthcare, our study is designed to describe African American men's experiences and cognitive perceptions, and to tap into patients and providers' subconscious assumptions, expectations, knowledge and practices. Based on our understanding of the structures and processes that hinder or enhance communication for African American men, we will identify prospects for intervention strategies with the potential to optimise the experience and contribute to improved outcomes.

\section{METHODS AND ANALYSIS \\ Study design}

From January 2017 through December 2020, we will conduct a longitudinal inductive qualitative descriptive study, specifically an ethnographic approach, with 25 newly diagnosed African American men and their 
providers to explore patient-provider interaction from PCa diagnosis through treatment decision. Ethnography is a qualitative research method central to sociology and anthropology and increasingly used in implementation science. ${ }^{40} 41$ It involves immersive, on-the-scene learning in natural situations. For this study, we take a multiperspective approach (including direct observation of people's behaviour and environments, and in-depth interviews with informants from varying perspectives) that is ideal for generating rich accounts of patientprovider interactions, ${ }^{42}{ }^{43}$ and details of patients' care experiences and providers' way of framing treatment options and delivering care. ${ }^{42} 44$ Importantly, observations illuminate dynamics that patients themselves may be unaware of and have difficulty surfacing. We use ethnography in accordance with the CHC framework to identify practices that lead to disparities, and to reveal how disparities are produced. ${ }^{45}$ In two very different safety net hospital settings, we will examine components of $\mathrm{CHC}^{38}$ including patients' health literacy, knowledge, control, trust, empowerment and interpersonal processes of care (eg, communication, decision-making and interpersonal style) using: (A) observations of clinic interactions in urology, radiation oncology and primary care when available; (B) in-depth interviews with patients and providers; (C) patient and provider surveys; and (D) field notes.

\section{Settings}

Our study is based in two safety net settings in California that vary in key system factors. At one public hospital (site A), patients are seen primarily by urology residents with attending physician supervision. In this setting, resident rotations afford little opportunity to establish ongoing trusting relationships. The second hospital (site B) is a public hospital with a large African American community. Three urologists provide ongoing care here and have a greater opportunity to establish long-term relationships with their patients.

\section{Study participants}

We propose a final study sample of 25 African American men with $\mathrm{PCa}-$ a number that is reasonable for achieving thematic saturation, and feasible within the study scope. As few as four to five patients can be appropriate when investigating qualitative, ethnographic cases in detail ${ }^{43}{ }^{46}$; and studies with multiple data sources (eg, observations and interviews) have achieved thematic saturation ${ }^{46} 47$ with three to nine cases. ${ }^{48-51}$ To achieve this final sample, we will recruit up to 80 African American men sent for biopsy, as we anticipate that $30 \%-40 \%$ of biopsies result in a PCa diagnosis, and should result in approximately 24-32 potential PCa cases.

Men who (A) self-identify as African American, (B) report they are able to speak English, $(\mathrm{C})$ are between the ages of 35 and 80 years, (D) do not have a history of PCa, (E) have an elevated PSA test, (F) are recommended for a prostate biopsy, and $(\mathrm{G})$ are not cognitively impaired will be invited to participate. We opted for recruitment prior to biopsy in order to capture subsequent patient-provider discussion at the time of a PCa diagnosis, establish patient rapport and to avoid both recruitment at a sensitive time (ie, during cancer diagnosis) and any potential for the recruitment procedures to prematurely reveal the $\mathrm{PCa}$ diagnosis. When introducing the study to eligible patients, we describe it as a research study about how doctors and patients talk about prostate biopsy results and next steps. There can be complicated information presented when receiving biopsy results. We want to learn if there are things we can do to improve their experience as a patient. Additionally, we inform patients that we will follow men to their appointments for up to 4 months, but potentially less, depending on plans for their healthcare in urology and what happens after the biopsy.

\section{Recruitment and consent}

Urologists, radiation oncologists and primary care providers at each site will be notified of the study via letter/email in advance of any recruitment and asked if they are willing to participate should a patient of theirs enroll in the study. We will offer in-person meetings to answer questions. The chiefs and medical director at both sites will encourage their colleagues' participation, as they provided enthusiastic support for the study proposal. Additionally, we received letters of support from current resident physicians, urologists on staff and a nurse practitioner from our partnering sites. We acknowledge there may be inadvertent coercive participation due to hierarchy; therefore, we will acquire verbal consent prior to original participation (eg, clinic observations) and again prior to providers completing a survey and in-depth interview (described below).

Patient identification and recruitment will vary slightly by clinic site. At site A, the study team will identify eligible men by reviewing upcoming clinic appointments scheduled in the urology clinic. During the clinic day, the provider seeing the eligible patient in urology will be notified of a patient's eligibility and will have an opportunity to decline participation (eg, due to known cognitive impairment). During the clinic visit for eligible patients, the provider asks the patient if it is okay for a researcher to come and talk to them about a study. If the patient gives permission, we then approach the eligible patient for recruitment, verbally introduce the study, review the printed consent form with the patient, answer questions and obtain written informed consent. If a patient indicates he needs more time to consider participation, we will provide our telephone number so he can call to ask more questions, and we will get his permission to follow-up with him at his next appointment (eg, prior to the prostate biopsy). Our consenting process will be repeated at the next appointment. At site $\mathrm{B}$, the chief of urology will identify eligible patients who are scheduled for upcoming prostate biopsy appointments, and will inform our team. Similarly, we then meet eligible patients at their scheduled biopsy appointment to verbally introduce the study, review the printed consent form with the 
patient, answer questions and obtain written informed consent prior to biopsy. If a patient at this site needs more time to consider participation, we will provide our telephone number and get his permission to follow-up with him prior to his appointment to receive biopsy results, to obtain written informed consent. If a patient is accompanied by a spouse, partner, caregiver or support person, we will obtain verbal informed consent from this companion, as they may be present during study procedures.

\section{Procedures and measures}

On enrolment, we will collect sociodemographic data from patients, including detailed contact information (phone, email, secondary contact person) for optimal retention. Standard urology protocol requires that results are delivered in person whether benign or malignant, usually within 1-2 weeks after biopsy. A member of our team (the principal investigator or research assistant) will be present to observe and audio record appointments that distribute biopsy results (eg, diagnosis appointment). Men who are not diagnosed with PCa will be thanked for their time and released from the study. We will be transparent that they are no longer needed for this study as they will not have any follow-up appointments in urology for PCa screening; however, we will add that if they return for a repeat biopsy we will re-engage them. Men without a PCa diagnosis will not be included in the final study sample $(n=25)$. Patients diagnosed with $\mathrm{PCa}$ will be followed at multiple clinic appointments relevant to PCa care until a treatment decision has been made (up to 2 months; see figure 1). We will conduct observations of multiple clinic visits, two in-depth interviews and up to four surveys with patients, all corresponding with scheduled clinic appointments for patient convenience and for retention. While data will not purposefully be collected from patients' companions (eg, spouse, partner, caregiver or support person) their comments will be captured on audio recordings of observed clinic appointments and included in analyses if relevant. We will also conduct one in-depth interview and survey with each treating provider seeing enrolled patients.

\section{Direct clinic observations}

Direct observation is used to study processes, procedures, activities or people in their lived environment. ${ }^{52}$ We will observe outpatient clinic appointments in urology, radiation oncology and medical oncology for patients newly diagnosed with PCa and considering treatment options. Due to the importance of coordination in high-quality cancer care ${ }^{8}$ and the active role primary care providers often play in cancer care,$^{53}$ we will also observe primary care provider appointments of enrolled patients. During clinic appointments, we will accompany enrolled patients as they see PCa specialists and primary care providers, remaining present but silent, audio recording the encounter and taking notes. On arrival, providers will be informed (or reminded) about the study purpose (eg, following patients newly diagnosed with $\mathrm{PCa}$ ) and the observer (lead author, NRP) will remain a constant fixture in the urology clinic throughout the study period as a regular member of the clinic team. Deviation from providers' normal behaviour is less likely when observation is sustained for an extended period of time. ${ }^{54}$ During direct observations, we will also take field notes ${ }^{55}$ to capture the substance of what was done and said, non-verbal behaviours (eg, eye contact, forward lean to indicate attentiveness and nodding to indicate understanding) and any other notable aspects of the appointment, patient or provider.

\section{Patient interviews and surveys}

Once patients are consented and enrolled, we will administer a baseline survey shortly after diagnosis that captures sociodemographic characteristics, health literacy, ${ }^{56}$

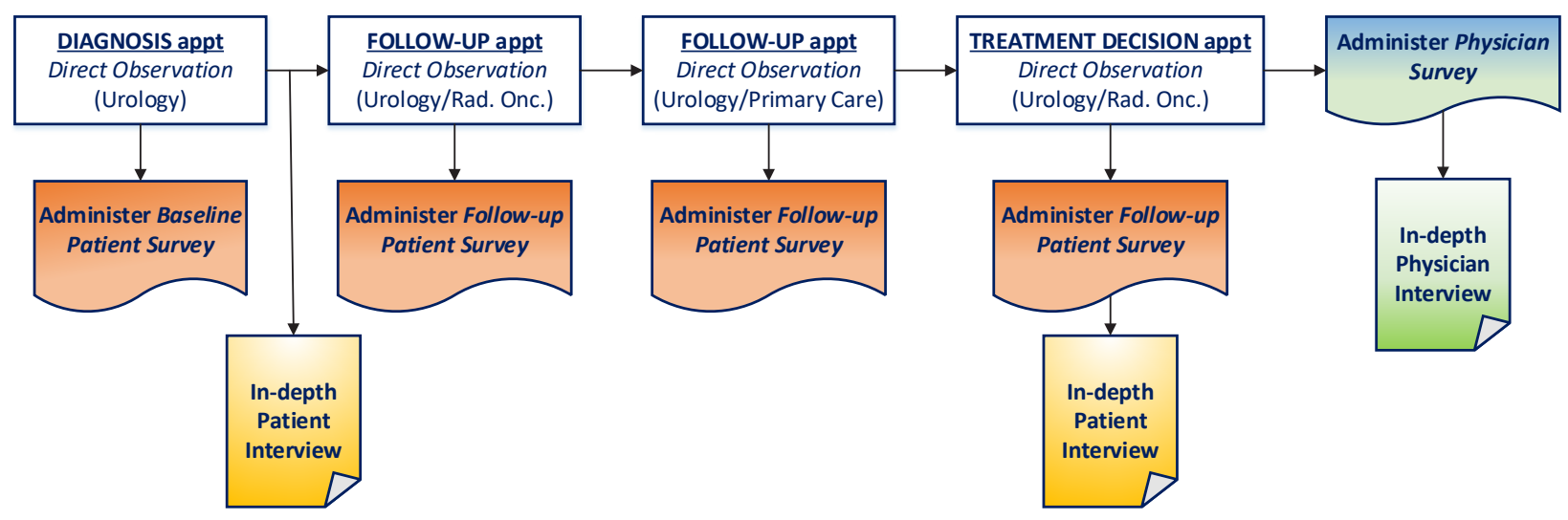

Figure 1 Data collection procedures. 
satisfaction with care $^{57}$ and interpersonal processes of care (eg, hurried communication, patient-centred decision-making, compassionate, and so on) ${ }^{58} \mathrm{Up}$ to three additional follow-up surveys will be completed after each clinic appointment that assesses communication, ${ }^{59}$ satisfaction with care ${ }^{57}$ perceived personal control ${ }^{60}$ and financial need. Repeated surveys can offer unique insights into the changing clinical portrait of patients' experience across various phases of cancer care ${ }^{61}$ For example, a patient's perception of patient-centred communication may improve (eg, better score) as they have repeated appointments during the decision-making process. To reduce literacy burden, we will verbally administer the survey that includes 46 questions; and each patient will receive a $\$ 15$ gift card immediately after completing each survey.

We will conduct two in-depth interviews with patients within 3 weeks of their observed diagnosis and treatment decision appointments. These interviews will take place in a private location at each clinic site, at a time convenient for patients (eg, patient already has a clinic appointment scheduled or patient is willing to return to the clinic at a convenient time). Patients will be asked to discuss their experience with the healthcare system and their current visits. We will then guide patients to reflect on what they know and understand from the clinical discussions about their diagnosis, treatment options and the decisionmaking process, and explore how these affect their interactions with the healthcare team, choice of treatment and their social support network. Parts of recorded observations will be replayed during the interview to aid in recall. Such 'stimulated recall' provides powerful and needed retrieval cues as clinical interactions are complex and conversation can be extensive. ${ }^{62-64}$ If a participant seems distressed or upset during any part of the research process, we will proactively offer to take a break, resume the interview at another time or terminate the observation or interview altogether. If needed, participants will also be immediately referred to a psychologist or social welfare professional for counselling. During the last in-depth interview, we will also ask patients how they felt about being part of this study, including being observed and recorded. Patients will receive a $\$ 35$ gift card immediately after completing each interview. If all surveys (up to 4 ) and interviews (2 total) are completed, patients will receive a total of $\$ 130$ for study participation.

\section{Provider interviews and surveys}

Once all patient data collection is complete, each provider who is involved in treatment decision-making of enrolled patients will complete a brief survey that captures sociodemographic characteristics, years since completing medical training (eg, residency), medical specialty, communication self-efficacy ${ }^{65}$ and health communication skills training received. Providers will then complete an in-depth interview. This timing is designed to avoid providers changing their care and communication practices based on their exposure to various survey and interview questions, and scenarios. We will ask providers about their strategies for communicating a PCa diagnosis, treatment options, potential side effects and decisionmaking; if and how they adapt their communication style for patient literacy; their expectations of patient involvement in decision-making; other people they believe should be involved in decision-making; impediments to treatment decision-making; and any reflections or relevant past experiences they have treating African American patients with PCa. Portions of recorded clinic observations will be replayed during the interview to elicit discussion and for recall, when relevant. ${ }^{62-64}$ We will also ask providers about their perceptions of the extent to which the observer may have impacted the degree of engagement or content of the patient-provider conversation and dynamic. Providers will receive a $\$ 75$ gift card for completing the interview, which we feel is reasonable given lower response rates relative to the general population. ${ }^{66}$ We recognise this incentive may not motivate providers to participate and they may decline the gift card; however, we know that some providers may appreciate the incentive to share treats with their team/office.

\section{Analysis plan}

All direct observations and qualitative interviews will be audio-recorded and transcribed verbatim, and imported into Dedoose software for coding, searching and retrieving coded text and audio. Dedoose is a crossplatform application for analysing qualitative and mixed methods research with text, audio, spreadsheet data and more, and is efficient for team coding. ${ }^{67}$ Field notes will also be typed up and imported into Dedoose. Analysis will be led by NRP with guidance by JKS and RJP; the entire team will be involved in data interpretation through sharing of emergent findings and theme summaries for discussion and feedback.

Using standard techniques based on grounded theory, ${ }^{68-70}$ we will follow an iterative process of deductive (eg, concept driven, based on constructs) ${ }^{71}$ and inductive (eg, text driven, allow themes to emerge $)^{71} 72$ thematic content analysis that involves examining and re-examining actions and meanings contained in the data to develop more refined codes and themes from all observations, field notes and in-depth interviews. This includes becoming immersed in the data through reading and re-reading, line-by-line coding and team discussions; finalising a codebook for significant concepts, and subsequent coding of themes and concepts; and identifying associations.

We will first develop a preliminary codebook of deductive codes (eg, provider inviting questions, patient interjecting questions, provider speaking over patient) based on $\mathrm{CHC}^{38}$ that includes definitions of codes and examples which will be organised by data sources (observations, patient interviews, provider interviews). Direct observations and patient interviews will enable us to explore (1) how the dynamics of CHC play out from the patient's perspective; (2) how the provider's approach and other 
system characteristics impact key relational components of obtaining care and decision-making; and (3) how health literacy and culture may influence various aspects of the patient's experience. Central to CHC is the concept of power dynamics-does the provider take steps to share power with the patient, or does she/he limit patient access to power by speaking past the patient or dominating the direction of the conversation? We will identify salient dimensions of patient trust and power/powerlessness, and power-sharing dynamics in clinical interactions. In addition, components of patient-provider communication and decision-making will be used to analyse type of communication, roles in communication and decisionmaking process among patients and providers. ${ }^{73-75}$ For example, to what extent are providers engaging (or NOT engaging) in directive or instructive communication (eg, provider focuses on what to do without asking opinions) or shared communication (eg, provider shares information adapting to patients' needs and preferences)? Similarly, what are patients' preferences for decisional support? To what extent are patients engaging in communication and decision-making that is passive (eg, patient listens to options passively taking in information), active on demand (eg, patient does not voluntarily intervene in communication, but will if invited by the doctor) or active (eg, patient is involved in exchange of information-asks questions, expresses concerns, raises issues, and so on) $?^{73}$ Importantly, patients vary in how they want to be involved in discussing and selecting treatments. Not all patients desire to take an active role in decisionmaking, but rather prefer a passive or 'delegators' role. ${ }^{76}$ We will also explore who patients want to involve in their care and decision-making. Does the patient feel confident and express his needs or concerns? Do the patient and provider share knowledge, information, needs and concerns in order to arrive at a decision together? How do patients express concerns about their masculine identity? How might their perceived threat to masculinity have an impact on communication with family members, friends and healthcare providers? How might patient's perspective on masculinity influence treatment decision-making? We will note the range of variation and attempt to explain what accounts for those differences. To follow, inductive codes will be generated that reflect additional insights beyond $\mathrm{CHC}$ and models of communication, ${ }^{1}$ and these new codes will be added to the codebook.

Two team members experienced in qualitative analysis will independently code three transcripts from clinic observations to see how the deductive codes work and what inductive codes arise. They will meet regularly to discuss coding strategies, and compare results in order to reach consensus about assigning a particular code to a given quote or text. This process will help populate and revise the codebook. The two researchers will repeat this process with three patient interviews, then three provider interviews, and three field notes, constantly going through an iterative process until we reach consistency. If needed, a third senior team member will be included in the discussion to resolve any disagreement between the two coders. Once the codebook is final, the researchers will code the remaining transcripts and work collaboratively to develop categories and identify themes that emerge. On reaching consensus on emerging themes, we will produce summaries of findings for discussion with the full research team.

These data will represent different perspectives. Given the multiple data sources (observations and interviews) and respondents (patients and providers), we anticipate some codes and themes are going to come up in one data source, but not another (eg, present in patient interviews, but absent in provider interviews); and some might come up in multiple or all data sources. To inform data integration and triangulation of multiple data sources and respondent groups (patients vs providers), we will note where codes are present versus missing in various data sources and groups, and explore convergence, complementarity and heterogeneity. We will also examine notable changes or evolution in codes and themes over time, as patients progress from diagnosis to treatment decision. To synthesise our multiple forms of qualitative data, we will follow a modified version of a six-step triangulation protocol developed by Farmer et al. ${ }^{77}$ The first step, sorting, refers to identifying the key themes in each data source (ie, observations, patient interviews, provider interviews and field notes) and respondent group (ie, patients and providers) to compare similarities and differences for the presence (or absence) and frequency, meaning and examples. Then we will code the degree and type of convergence-including agreement (full agreement between sources and respondents), partial agreement (agreement on one but not another theme), silence (theme is absent in one) and heterogeneity (variation between elements of comparison). ${ }^{77}$

All survey data will be analysed using Statistical Package for the Social Sciences (SPSS) software V.25. Separate summary statistics for patients and providers will be calculated for categorical (number, percent, minimum and maximum) and continuous (mean, median, SD and range) variables to describe the study population. Repeated measures (eg, patient-centred communication scores) will be assessed for changes or improvements over time. Survey results will also be reviewed in the context of the qualitative data, which may confirm, conflict or elucidate survey data.

\section{Potential challenges}

Recruitment may be challenging due to insufficient numbers of African American patients. If enrolment lags at one site, we will (A) increase the sample size at the more productive site, (B) extend recruitment, and (C) add a third clinical site where our team has established prior research partnerships. We recognise physicians may serve as 'gatekeepers' and could bias and impact recruitment efforts. ${ }^{78}$ In light of key urology leadership supporting our research proposal, we do not anticipate this being a problem, but we will monitor it closely. For 
any patients who are not comfortable being observed, we will leave the audio recorder on in the room and step out of the clinic appointment. This strategy is also useful to overcome scheduling issues, in which a team member may not be available to attend all enrolled patients' clinic appointments. In this case, the provider will be asked to audio record the clinic visit with an extra audio recorder stored at the site.

\section{Patient and public involvement}

Our team's extensive work in community engagement in PCa disparities over the past 6 years contributed to the development and ideas of this study. Specifically, members of a PCa support group led by one of our team members (NRP), which was started 3 years before this study was funded, influenced the launch of this study, ideas for potential interventions and a robust plan to engaging community stakeholders. However, patients were not officially involved in the design, recruitment of conduct of this study. Once data collection is complete, we will convene a Community Advisory Team (CAT) of African American PCa survivors, some of whom may have been enrolled in this study, and other community members engaged in various initiatives, including the Prostate Health Support Group and the Prostate Cancer Action Network (of the San Francisco Cancer Initiative (SF CAN) (described below)). The CAT will meet monthly to interpret data collected in this study, and contribute to the development of the next phase of intervention development to ensure cultural fit. We will also disseminate study results, in lay language, beyond the CAT to the support group and the Prostate Cancer Action Network.

\section{ETHICS AND DISSEMINATION}

This study was approved by the University of California San Francisco Institutional Review Board (16-20126) and the Alameda Health System Institutional Review Board (IRB17-03311A). Our study is designed to examine the ways in which PCa diagnoses and treatment options are communicated to African American patients, and patients' treatment decision-making. The longitudinal design of this study will yield a rich understanding of the experiences of African American patients with PCa from diagnosis through treatment decision-making, and identify gaps in communication where additional support may be necessary. The collection of multimodal qualitative approaches (observations and interviews) and informants, and quantitative data will facilitate a more holistic perspective. This comprehensive view will inform next steps in intervention development, in which community stakeholders will be engaged as cocreators (eg, collaborators) in interpreting ethnographic findings for maximum cultural fit and impact. Research findings from synthesising all of the integrated information from this study will inform intervention development with the goal to help aid communication between patients and providers and close key gaps identified from our results.
Following study completion, findings will be written up for submission to scientific conference presentations and peer-reviewed journals for publication. A summary of findings will be presented to collaborating clinical sites and providers involved in the study. As the next pilot intervention phase of this research will include community engagement, we will also prepare a lay summary for dissemination to study participants and a communitybased patient advisory board, and provide an opportunity for feedback and discussion on the next steps of this research.

This research will also inform current programmes in the community-specifically the SF CAN Prostate Cancer Task Force. SF CAN is a city-wide initiative to reduce cancer-related morbidity and mortality by harnessing the collective impact ${ }^{79}$ across major healthcare systems, community coalitions and community members. ${ }^{80}$ The SF CAN Prostate Cancer Task Force ${ }^{81}$ is working collectively to support healthcare providers and engage African American men regarding decisions for the detection and treatment of PCa, to decrease the overtreatment of lowrisk disease and undertreatment of aggressive disease and eliminate the disparity in PCa mortality for African Americans. Activities include a quality collaborative, continuing medical education, community engagement and education, navigation/coaching and survivorship support.

\section{Author affiliations \\ ${ }^{1}$ Division of General Internal Medicine at San Francisco General Hospital, Department of Medicine, University of California San Francisco, San Francisco, California, USA \\ ${ }^{2}$ Center for Vulnerable Populations, San Francisco General Hospital, University of California San Francisco, San Francisco, California, USA \\ ${ }^{3}$ Helen Diller Family Comprehensive Cancer Center, University of California San Francisco, San Francisco, California, USA \\ ${ }^{4}$ Department of Social and Behavioral Sciences, School of Nursing, University of California San Francisco, San Francisco, California, USA \\ ${ }^{5}$ Division of General Internal Medicine, Department of Medicine, University of California San Francisco, San Francisco, California, USA \\ ${ }^{6}$ Division of Urology, Highland Hospital, Oakland, California, USA \\ ${ }^{7}$ Department of Urology, University of California San Francisco, San Francisco, California, USA \\ ${ }^{8}$ Department of Epidemiology and Biostatistics, University of California San Francisco, San Francisco, California, USA}

\section{Twitter Nynikka R Palmer @PalmerNynikka}

Acknowledgements We acknowledge the scholars of the UCSF Center for Aging in Diverse Communities and the UCSF K Scholars Program for their feedback during NRP's work-in-progress presentations. We thank Brittany Campbell for manuscript formatting assistance.

Contributors NRP is the overall project leader and the grant holder with responsibility for the design and execution of the protocol. All authors contributed to the initial conception, the design of the study and the development and refinement of the protocol. NRP led the development of the initial study protocol, which RJP, CPK, DS, JKS, BNB and SDB reviewed and provided feedback. NRP led the development of instruments, interview guides and schedule for data collection, with input from all coauthors. RJP, BNB and JKS provided expertise on recruitment and data collection processes and systems for collecting data from medical records. NRP drafted this manuscript and all coauthors provided critical feedback. All authors read and approved the final version of the manuscript.

Funding This work was supported by the National Institute on Aging of the National Institutes of Health under grant number P30AG015272 (University of California San Francisco, Center for Aging in Diverse Communities); the National 
Center for Advancing Translational Sciences of the National Institutes of Health under grant number KL2TR001870; and the National Cancer Institute of the National Institutes of Health under grant number K01CA211965.

Disclaimer The content is solely the responsibility of the authors and does not necessarily represent the official views of the National Institutes of Health. The funding bodies played no role in the design of the study and collection, analysis, and interpretation of data and in writing the manuscript.

Competing interests None declared.

Patient and public involvement Patients and/or the public were involved in the design, or conduct, or reporting, or dissemination plans of this research. Refer to the Methods section for further details.

Patient consent for publication Not required.

Ethics approval This study was approved by the University of California San Francisco Institutional Review Board (16-20126) and the Alameda Health System Institutional Review Board (IRB17-03311A). Written informed consent is obtained from all patient participants. Verbal consent is obtained from all providers of enrolled patients, as written consent was waived by the University of California San Francisco Institutional Review Board.

Provenance and peer review Not commissioned; externally peer reviewed.

Open access This is an open access article distributed in accordance with the Creative Commons Attribution Non Commercial (CC BY-NC 4.0) license, which permits others to distribute, remix, adapt, build upon this work non-commercially, and license their derivative works on different terms, provided the original work is properly cited, appropriate credit is given, any changes made indicated, and the use is non-commercial. See: http://creativecommons.org/licenses/by-nc/4.0/.

ORCID iD

Nynikka R Palmer http://orcid.org/0000-0002-5311-447X

\section{REFERENCES}

1 Epstein RM, Street RL. Patient-Centered communication in cancer care: promoting healing and reducing suffering. National Institutes of health, NIH publication No. 07-6225. Bethesda, MD, 2007.

2 American Cancer Society. Cancer Facts \& Figures for African Americans 2016-2018. Atlanta: American Cancer Society, 2016.

3 Siegel RL, Miller KD, Jemal A. Cancer statistics, 2019. CA Cancer J Clin 2019;69:7-34.

4 Chornokur G, Dalton K, Borysova ME, et al. Disparities at presentation, diagnosis, treatment, and survival in African American men, affected by prostate cancer. Prostate 2011;71:985-97.

5 Jayadevappa R, Chhatre S, Johnson JC, et al. Variation in quality of care among older men with localized prostate cancer. Cancer 2011;117:2520-9.

6 Presley CJ, Raldow AC, Cramer LD, et al. A new approach to understanding racial disparities in prostate cancer treatment. $J$ Geriatr Oncol 2013;4:1-8.

7 Mahal BA, Aizer AA, Ziehr DR, et al. Trends in disparate treatment of African American men with localized prostate cancer across national comprehensive cancer network risk groups. Urology 2014;84:386-92.

8 Committee on Improving the Quality of Cancer Care: Addressing the Challenges of an Aging Population, Board on Health Care Services, Institute of Medicine. Delivering high-quality cancer care: charting a new course for a system in crisis. Levit L, Balogh E, nass S, Ganz PA, editors. Washington (DC): National Academies Press (US), 2013.

9 Reichard CA, Hoffman KE, Tang C, et al. Radical prostatectomy or radiotherapy for high- and very high-risk prostate cancer: a multidisciplinary prostate cancer clinic experience of patients eligible for either treatment. BJU Int 2019.

10 Mohler JL, Antonarakis ES, Armstrong AJ, et al. Prostate cancer, version 2.2019, NCCN clinical practice guidelines in oncology. J Natl Compr Cancer Netw 2019;17:479-505.

11 Litwin MS, Tan $\mathrm{H}-\mathrm{J}$. The diagnosis and treatment of prostate cancer: a review. JAMA 2017;317:2532-42.

12 van Stam M-A, van der Poel HG, van der Voort van Zyp JRN, et al. The accuracy of patients' perceptions of the risks associated with localised prostate cancer treatments. BJU Int 2018;121:405-14.

13 Gamble VN. Under the shadow of tuskegee: African Americans and health care. Am J Public Health 1997;87:1773-8.

14 Halbert $\mathrm{CH}$, Weathers B, Delmoor E, et al. Racial differences in medical mistrust among men diagnosed with prostate cancer. Cancer 2009;115:2553-61.
15 Rivers BM, August EM, Gwede CK, et al. Psychosocial issues related to sexual functioning among African-American prostate cancer survivors and their spouses. Psychooncology 2011;20:106-10.

16 Rivas C, Matheson L, Nayoan J, et al. Ethnicity and the prostate cancer experience: a qualitative metasynthesis. Psychooncology 2016;25:1147-56.

17 Campbell LC, Keefe FJ, McKee DC, et al. Masculinity beliefs predict psychosocial functioning in African American prostate cancer survivors. Am J Mens Health 2012;6:400-8.

18 Pedersen VH, Armes J, Ream E. Perceptions of prostate cancer in black African and black Caribbean men: a systematic review of the literature. Psychooncology 2012;21:457-68.

19 Imm KR, Williams F, Housten AJ, et al. African American prostate cancer survivorship: exploring the role of social support in quality of life after radical prostatectomy. J Psychosoc Oncol 2017;35:409-23.

20 Berkman ND, Sheridan SL, Donahue KE, et al. Low health literacy and health outcomes: an updated systematic review. Ann Intern Med 2011;155:97-107.

21 Song L, Mishel M, Bensen JT, et al. How does health literacy affect quality of life among men with newly diagnosed clinically localized prostate cancer? findings from the North Carolina-Louisiana prostate cancer project (PCaP). Cancer 2012;118:3842-51.

22 Wolf MS, Knight SJ, Lyons EA, et al. Literacy, race, and PSA level among low-income men newly diagnosed with prostate cancer. Urology 2006;68:89-93.

23 Ziehr DR, Mahal BA, Aizer AA, et al. Income inequality and treatment of African American men with high-risk prostate cancer. Urol Oncol 2015;33:18.e7-18.e13.

24 Mahal BA, Ziehr DR, Aizer AA, et al. Getting back to equal: the influence of insurance status on racial disparities in the treatment of African American men with high-risk prostate cancer. Urol Oncol 2014;32:1285-91.

25 Wang EH, Yu JB, Abouassally R, et al. Disparities in treatment of patients with high-risk prostate cancer: results from a populationbased cohort. Urology 2016;95:88-94.

26 Pollack CE, Bekelman JE, Epstein AJ, et al. Racial disparities in changing to a high-volume urologist among men with localized prostate cancer. Med Care 2011;49:1-1006.

27 Krimphove MJ, Fletcher SA, Cole AP, et al. Quality of care in the treatment of localized intermediate and high risk prostate cancer at minority serving hospitals. J Urol 2019:201:735-41.

28 McCaffery KJ, Smith SK, Wolf M. The challenge of shared decision making among patients with lower literacy: a framework for research and development. Med Decis Making 2010;30:35-44.

29 Kim SP, Knight SJ, Tomori C, et al. Health literacy and shared decision making for prostate cancer patients with low socioeconomic status. Cancer Invest 2001;19:684-91.

30 Wagland R, Nayoan J, Matheson L, et al. 'Very difficult for an ordinary Guy': factors influencing the quality of treatment decisionmaking amongst men diagnosed with localised and locally advanced prostate cancer: findings from a UK-wide mixed methods study. Patient Educ Couns 2019;102:797-803.

31 Rood JAJ, Nauta IH, Witte BI, et al. Shared decision-making and providing information among newly diagnosed patients with hematological malignancies and their informal caregivers: Not "onesize-fits-all". Psychooncology 2017;26:2040-7.

32 Gilbar R, Miola J. One size fits all? on patient autonomy, medical decision-making, and the impact of culture. Med Law Rev 2015;23:375-99.

33 Shay LA, Lafata JE. Understanding patient perceptions of shared decision making. Patient Educ Couns 2014;96:295-301.

34 Cuypers M, Lamers RED, de Vries M, et al. Prostate cancer survivors with a passive role preference in treatment decision-making are less satisfied with information received: results from the profiles registry. Urol Oncol 2016;34:482.e11-e18.

35 Palmer NRA, Tooze JA, Turner AR, et al. African American prostate cancer survivors' treatment decision-making and quality of life. Patient Educ Couns 2013:90:61-8.

36 Diefenbach MA, Dorsey J, Uzzo RG, et al. Decision-Making strategies for patients with localized prostate cancer. Semin Urol Oncol 2002;20:55-62.

37 Fowler FJ, McNaughton Collins M, Albertsen PC, et al. Comparison of recommendations by urologists and radiation oncologists for treatment of clinically localized prostate cancer. JAMA 2000;283:3217-22

38 Shim JK. Cultural health capital: a theoretical approach to understanding health care interactions and the dynamics of unequal treatment. J Health Soc Behav 2010;51:1-15.

39 Pasick RJ, Burke NJ, Barker JC, et al. Behavioral theory in a diverse Society: like a compass on Mars. Health Educ Behav 2009;36:11S-35. 
40 Atkinson P, Coffey A, Delamont S, et al. Handbook of ethnography. SAGE, 2001.

41 Sinead Ryan G. An introduction to the origins, history and principles of ethnography. Nurse Res 2017:24:15-21.

42 Leslie M, Paradis E, Gropper MA, et al. Applying ethnography to the study of context in healthcare quality and safety. BMJ Qual Saf 2014;23:99-105.

43 Reeves S, Kuper A, Hodges BD. Qualitative research methodologies: ethnography. BMJ 2008;337:a1020.

44 Hamilton H, Chou WS. The Routledge Handbook of language and health communication. Routledge, 2014.

45 Sinding C. Using institutional ethnography to understand the production of health care disparities. Qual Health Res 2010;20:1656-63.

46 Creswell JW. A Concise introduction to mixed methods research. SAGE Publications, 2014.

47 Guest G, Bunce A, Johnson L. How many interviews are enough?: an experiment with data saturation and variability. Field Methods 2006;18:59-82.

48 Unertl KM, Weinger M, Johnson K. Variation in use of informatics tools among providers in a diabetes clinic. AMIA Annu Symp Proc 2007:756-60.

49 Dhand A, Engstrom J, Dhaliwal G. How experienced community neurologists make diagnoses during clinical encounters. Neurology 2013;81:1460-6.

50 Carpenter-Song EA, Holcombe BD, Torrey J, et al. Recovery in a family context: experiences of mothers with serious mental illnesses. Psychiatr Rehabil J 2014;37:162-9.

51 Shershneva MB, Mullikin EA, Loose A-S, et al. Learning to collaborate: a case study of performance improvement CME. $J$ Contin Educ Health Prof 2008;28:140-7.

52 McCullough MB, Petrakis BA, Gillespie C, et al. Knowing the patient: a qualitative study on care-taking and the clinical pharmacist-patient relationship. Res Soc Adm Pharm 2016;12:78-90.

53 Klabunde CN, Ambs A, Keating NL, et al. The role of primary care physicians in cancer care. J Gen Intern Med 2009;24:1029-36.

54 Goodwin MA, Stange KC, Zyzanski SJ, et al. The Hawthorne effect in direct observation research with physicians and patients. J Eval Clin Pract 2017;23:1322-8.

55 Mulhall A. In the field: notes on observation in qualitative research. $J$ Adv Nurs 2003;41:306-13.

56 Wallston KA, Cawthon C, McNaughton CD, et al. Psychometric properties of the brief health literacy screen in clinical practice. $J$ Gen Intern Med 2014;29:119-26.

57 Jean-Pierre P, Fiscella K, Freund KM, et al. Structural and reliability analysis of a patient satisfaction with cancer-related care measure: a multisite patient navigation research program study. Cancer 2011;117:854-61.

58 Stewart AL, Nápoles-Springer AM, Gregorich SE, et al. Interpersonal processes of care survey: patient-reported measures for diverse groups. Health Serv Res 2007;42:1235-56.

59 Makoul G, Krupat E, Chang C-H. Measuring patient views of physician communication skills: development and testing of the communication assessment tool. Patient Educ Couns 2007;67:333-42.

60 Manne S, Glassman M. Perceived control, coping efficacy, and avoidance coping as mediators between spouses' unsupportive behaviors and cancer patients' psychological distress. Health Psychol 2000;19:155-64.

61 Thorne S, Oliffe JL, Stajduhar Kl, et al. Poor communication in cancer care: patient perspectives on what it is and what to do about it. Cancer Nurs 2013;36:445-53.

62 Elliott R. Interpersonal Process Recall (IPR) as a psychotherapy process research method. In: The psychotherapeutic process: a research Handbook. New York, NY, US: Guilford Press, 1986.

63 Larsen D, Flesaker K, Stege R. Qualitative interviewing using interpersonal process recall: investigating internal experiences during Professional-Client conversations. Int J Qual Methods 2008;7:18-37.

64 Kagan N, Krathwohl DR, Miller R. Stimulated recall in therapy using video tape: a case study. J Couns Psychol 1963;10:237-43.

65 Zachariae R, O'Connor M, Lassesen B, et al. The self-efficacy in patient-centeredness questionnaire - a new measure of medical student and physician confidence in exhibiting patient-centered behaviors. BMC Med Educ 2015;15:150.

66 Wiant K, Geisen E, Creel D, et al. Risks and rewards of using prepaid vs. postpaid incentive checks on a survey of physicians. BMC Med Res Methodol 2018;18:104

67 SocioCultural Research Consultants, LLC. Dedoose version 7.0.23 Available: https://www.dedoose.com/ [Accessed 17 Aug 2019].

68 Glaser BG, Strauss AL, Strutzel E. Discovery of Grounded theory; strategies for qualitative research. New York: Routledge, 1999.

69 Miles MB, Huberman AM, Huberman MA, et al. Qualitative data analysis: an expanded Sourcebook. SAGE, 1994.

70 Strauss AL. Qualitative analysis for social scientists. Cambridge University Press, 1987.

71 Graneheim UH, Lindgren B-M, Lundman B. Methodological challenges in qualitative content analysis: a discussion paper. Nurse Educ Today 2017;56:29-34.

72 Elo S, Kyngäs $\mathrm{H}$. The qualitative content analysis process. $J$ Adv Nurs 2008;62:107-15

73 Renzi C, Fioretti C, Oliveri S, et al. A qualitative investigation on patient Empowerment in prostate cancer. Front Psychol 2017;8:1215.

74 Emanuel EJ, Emanuel LL. Four models of the physician-patient relationship. JAMA 1992;267:2221-6.

75 Elwyn G, Frosch D, Thomson R, et al. Shared decision making: a model for clinical practice. J Gen Intern Med 2012;27:1361-7.

76 Flynn KE, Smith MA, Vanness D. A typology of preferences for participation in healthcare decision making. Soc Sci Med 2006;63:1158-69.

77 Farmer T, Robinson K, Elliott SJ, et al. Developing and implementing a triangulation protocol for qualitative health research. Qual Health Res 2006;16:377-94.

78 Thomas N, Plant S, Woodward-Nutt K, et al. Health care professionals' views of the factors influencing the decision to refer patients to a stroke rehabilitation trial. Trials 2015;16:577.

79 Kania J, Kramer M. Collective impact. Stanford Social Innovation Rev 2011;9:36-41.

80 Hiatt RA, Sibley A, Fejerman L, et al. The San Francisco cancer initiative: a community effort to reduce the population burden of cancer. Health Aff 2018;37:54-61.

81 San Francisco Cancer Initiative. Sf can prostate cancer Task force, 2016. Available: http://www.sfcancer.org/prostate-cancer [Accessed 16 Aug 2019]. 